\title{
Sharman, J.C.: Empires of the Weak. The Real Story of European Expansion and the Creation of the New World Order, 216 S., Princeton UP, Princeton, NJ/Oxford 2019.
}

\author{
Jürgen Osterhammel \\ Online publiziert: 19. Februar 2020 \\ (C) Der/die Autor(en) 2020
}

In die endlose Debatte um die Ursprünge der europäischen Weltdominanz in der Neuzeit ist in den letzten Jahren etwas Ruhe eingekehrt. Die Diskussion ist vorwiegend auf dem Gebiet der vergleichenden Wirtschaftsgeschichte über die Ursprünge einer globalen Wohlstandsschere geführt worden. Hier standen (und stehen) sich, grob kategorisiert, vier Lager gegenüber: die Kulturalisten, die Anhänger der Neuen Institutionenökonomie, die Umwelthistoriker, schließlich die Vertreter eines staatszentrierten Ansatzes. Bis heute hat man sich zwischen diesen Positionen kaum angenähert; eine halbwegs einvernehmliche Lösung ist nicht in Sicht. Das Thema der zeitweiligen militärischen und politischen Übermacht Europas über den Rest der Welt ist mit dieser ökonomischen Debatte selten systematisch verknüpft worden. Die historischen Soziologen, die sich dafür interessieren (der bekannteste von ihnen dürfte Michael Mann sein), haben die Wirtschaft nur am Rande im Blick.

Das ist auch so bei Jason Sharman, Professor für Internationale Beziehungen in Cambridge. Ohne sich auf die Kontroverse um die great divergence $\mathrm{zu}$ beziehen, fragt er in ausschließlich machtpolitischer Perspektive nach den Ursprüngen der heutigen ,neuen Weltordnung“. Was er darunter versteht, wird nicht näher erläutert. Offenbar das heute Übliche: Niedergang Europas, Aufstieg Chinas. Die Weichenstellungen zur Gegenwart sieht Sharman in der Frühen Neuzeit. Das 19. und das 20. Jahrhundert interessieren ihn wenig; die Gegenwart ergibt sich pfadabhängig aus dem 16. bis 18. Jahrhundert.

Nun gibt es schon einen ganzen Chor von, je nachdem, Klage- oder Freudengesängen über den Niedergang Europas. Was hört man bei Sharman Neues? Er hat sich gründlich in die englischsprachige Literatur zur europäischen Expansion er benutzt diesen Begriff ironisch - eingearbeitet und ist dabei als Politikwissenschaftler zu der pfiffigen Erkenntnis gelangt, die Historiker würden ungeschickt mit rational-choice-Annahmen und darwinistischen Selektionsmodellen hantieren, ohne es selbst zu merken. Dem möchte er einen Primat „kultureller Faktoren“ entgegenstellen, belässt es dann aber bei dieser vagen Ankündigung. Offenbar sind zählebige Weltbilder gemeint.

J. Osterhammel $(\bowtie)$

Albert-Ludwigs-Universität Freiburg im Breisgau, Freiburg i.Br., Deutschland

E-Mail: juergen.osterhammel@ frias.uni-freiburg.de 
Sharman hat keine eigene Theorie vorzuschlagen, sondern verfolgt mit diesem Buch, das trotz seiner Kürze entnervend repetitiv ist, dekonstruierende Absichten. Sein polemischer Drang ist so stark, dass man seinen Darstellungen historischer Sachverhalte - beispielsweise der spanischen Eroberung Amerikas, des Aufbaus eines portugiesischen Überseereiches, der osmanischen Militärorganisation - nicht ohne Weiteres trauen kann. Er fasst seine Lesefrüchte nicht ungeschickt zusammen, jedoch stets auf seine obsessiv verfolgte eigene Argumentationslinie hin getrimmt. Wenn dies eine „real history“ der europäischen Expansion sein soll, dann eine überaus subjektive.

Die Polemik geht in zwei Richtungen. Erstens attackiert Sharman die These von einer „militärischen Revolution“ im frühneuzeitlichen Europa, wie sie Geoffrey Parker 1988 in seinem Buch „The Military Revolution“ ausgeführt hat. Sharman scheint sich vor allem darüber aufzuregen, dass diese These von seinen Kollegen in den International Relations ungeprüft als Dogma übernommen wird. Er bestreitet sie keineswegs für Europa. Aber er findet, dass sie die militärische Überwältigung amerikanischer, asiatischer und afrikanischer Staaten und Gesellschaften nicht erklären kann. Im Grunde hätten die Europäer vor 1800 selten gesiegt. Sie hätten sich den vorgefundenen Machtstrukturen angepasst oder sogar unterworfen.

Dies würde fast niemand in der Frühneuzeitforschung heute mehr bestreiten. Aber Sharman geht mit seinen tendenziösen Arrangements aus der Forschungsliteratur zu weit, und er schlägt logische Purzelbäume, um seine These vom „schwachen“Europa zu untermauern. Dass die Europäer in Mexiko oder Indien kleinere Kampfverbände einsetzten, als die Theorie der militärischen Revolution angeblich postuliert, ist weniger interessant als die Tatsache, dass sie am Ende stabile Kolonialherrschaft errichteten. Wenn der Autor nicht gerade offene Türen einrennt, übt er sich in wenig plausiblen Scheinbeweisen.

Zweitens unterstellt Sharman „den“ Historikern einen unausrottbaren eurozentrischen „Triumphalismus“, bleibt aber stichhaltige Nachweise dafür schuldig, dass solche Positionen heute noch seriös vertreten werden. Sie werden es nicht. Seine eigene Interpretationslinie ist a priori so anti-eurozentrisch wie nur möglich. Während der amerikanische Althistoriker Ian Morris in seinem Bestseller ,Why the West Rules - For Now" (2010) noch eine Geschichte von Aufstieg und Fall Europas erzählt hatte, geht Sharman - ohne das immer wieder angekündigte neue „Material“ vorzulegen - so weit, zu bestreiten, dass es jemals eine genuine europäische Expansion gegeben habe. Ausnahmslos und immer seien die Europäer schwächer gewesen, als „man“ (wer, bitte?) bisher geglaubt habe.

Soweit zur Frühen Neuzeit. Was aber ist mit dem ,imperialen Zeitalter“ und der Dekolonisation, die sich beide kaum leugnen lassen? Dazu äußert sich Sharman nur sehr knapp und dies mit einem schier unglaublichen Taschenspielertrick: Man müsse einen kurzsichtigen „bias of time“ überwinden; in langfristiger Betrachtung sei der moderne Kolonialismus nur eine flüchtige Episode gewesen und deshalb nicht der Beachtung wert. Das soll er mal im Globalen Süden erzählen!

Sharman entscheidet sich niemals, ob er primär empirisch oder ideologisch argumentieren soll, anders gesagt: ob er Geoffrey Parker, einem der weltweit renommiertesten Frühneuzeitexperten, vorwerfen will, ein schlechter Historiker oder ein engstirniger Eurozentrist zu sein. So oder so verstrickt sich die politische Botschaft 
des Buches in einen Widerspruch: Wenn Europa tatsächlich nie etwas Anderes war als ein aufgeplusterter Schwächling, wenn mangels Aufstieg von keinem Niedergang die Rede sein kann, dann erübrigt sich die laute Empörung über Eurozentrismus. Denn dann kann man mit Europa nur retrospektives Mitleid haben.

Berühmte Wissenschaftler preisen auf dem Cover die Originalität dieses Buches. Zu Recht, folgt man der zweiten Wortbedeutung, die der Duden kennt: ,eigenartig“. Es ist ,perversely original“, ohne jede Beweiskraft und ein Tiefpunkt in der Debatte über die Vorgeschichte der heutigen internationalen Ordnung.

Funding Open Access funding provided by Projekt DEAL.

Open Access Dieser Artikel wird unter der Creative Commons Namensnennung 4.0 International Lizenz veröffentlicht, welche die Nutzung, Vervielfältigung, Bearbeitung, Verbreitung und Wiedergabe in jeglichem Medium und Format erlaubt, sofern Sie den/die ursprünglichen Autor(en) und die Quelle ordnungsgemäß nennen, einen Link zur Creative Commons Lizenz beifügen und angeben, ob Änderungen vorgenommen wurden.

Die in diesem Artikel enthaltenen Bilder und sonstiges Drittmaterial unterliegen ebenfalls der genannten Creative Commons Lizenz, sofern sich aus der Abbildungslegende nichts anderes ergibt. Sofern das betreffende Material nicht unter der genannten Creative Commons Lizenz steht und die betreffende Handlung nicht nach gesetzlichen Vorschriften erlaubt ist, ist für die oben aufgeführten Weiterverwendungen des Materials die Einwilligung des jeweiligen Rechteinhabers einzuholen.

Weitere Details zur Lizenz entnehmen Sie bitte der Lizenzinformation auf http://creativecommons.org/ licenses/by/4.0/deed.de.

\section{Adi, Hakim: Pan-Africanism. A History, 312 S., Bloomsbury, London/New York 2018.}

\section{Arno Sonderegger}

Online publiziert: 14. Februar 2020

(C) Gesellschaft zur wissenschaftlichen Förderung politischer Literatur e.V. and the Author(s) 2020

Der britische Historiker Hakim Adi hat kürzlich eine umfassende Geschichte des Panafrikanismus vorgelegt. Das Phänomen selbst entzieht sich, wie er einleitend klar macht, aus mehreren Gründen jeder einfachen Definition (S. 2-5). Zunächst liegt das daran, dass es sich durch die Zeiten hindurch in vielfältigen Formen von Aktivitäten und Organisationsweisen äußerte und eine Menge unterschiedlicher und

\footnotetext{
A. Sonderegger $(\bowtie)$

Wien, Österreich

E-Mail: arno.sonderegger@univie.ac.at
} 